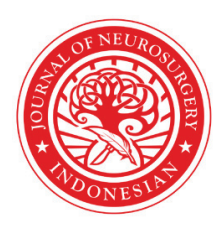

\title{
Extradural hematoma: Experience in achieving zero mortality of children patient in a single center
}

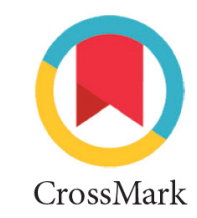

\author{
Lalithambigai Gannison ${ }^{\text {* }}$, Senthil Kumar Rajapathy ${ }^{1}$, Prabu Rau Sriram", \\ Sofan Zenian' ${ }^{1}$ Ananda Arumugam¹, Pulivendhan Sellamuthu
}

\section{ABSTRACT}

Introduction: Extradural hematoma (EDH), a common sequela of traumatic brain injury, can lead to mortality and disability if there is a delay in identifying the pathology and subsequent management. In the 1920s, the mortality rate was reported as $86 \%$ and improved to $10 \%$ over the new millennium. We review our EDH cases to identify how we achieved zero mortality, which is the ultimate goal of EDH management.

Methods: We retrospectively reviewed all traumatic EDH cases seen in the specialized pediatric neurosurgery department of Sabah Women \& Children Hospital from January 2013 to August 2018. Patients' case records, operative notes and radiological images were tracked and reviewed. A total of 101 cases were identified.

Results: We had 101 patients ranging from the age of 9 months to 12 years. Fifty-eight patients (58.41\%) were males, and 42 were females (41.59\%). A total of 37 patients (36.3\%) were treated surgically, and 64 patients (63.7\%) were treated conservatively.
About 92 patients (91.09\%) presented with supratentorial EDH and 9 patients (8.91\%) had infratentorial EDH. Skull fractures were present in 47 children (46.5\%). In the pediatric age group, fall from heights was the most common cause. We achieved zero mortality among our patients who were treated both surgically and conservatively.

Conclusion: EDH is a neurotrauma emergency that can be lethal, yet it is the most rewardingly responsive lesions treated by neurosurgeons. Early diagnosis, prompt referrals, early surgical intervention when indicated, and good intensive care remain the paramount facets towards achieving zero mortality in EDH. This study proves that our results are comparable to those seen in other neurosurgical centers throughout the developed world. An institutional protocol for EDH may ensure that the ultimate goal of towards 'zero mortality' could be achieved systematically and consistently.

Keywords: conservative, extradural hematoma, pediatric, surgical, zero mortality

Cite This Article: Gannison, L., Rajapathy, S.K., Sriram, P.R., Zenian, S., Arumugam, A., Sellamuthu, P. 2020. Extradural hematoma: Experience in achieving zero mortality of children patient in a single center. Indonesian Journal of Neurosurgery 3(1): 20-23. D0I: 10.15562/ ijn.v3i1.78

'Department of Neurosurgery Sabah, Kota Kinabalu, Malaysia

*Corresponding author: Lalithambigai Gannison; Department of Neurosurgery Sabah, Kota Kinabalu, Malaysia; lali_gannison@yahoo.com
Received: 2019-08-11 Accepted: 2019-09-01 Published: 2020-04-09

\section{INTRODUCTION}

Extradural hematoma (EDH), a collection of blood between the skull and the dura mater due to various causes of bleed, is a common sequela of head injury. ${ }^{1-3}$ It is rare in children and can be fatal if not treated expeditiously. ${ }^{4}$ As the nature of extradural hematoma is extracerebral, all patients should be saved. This pathology becomes lifethreatening when it causes irreversible damage due to brain herniation and intracranial hypertension., Computed tomography (CT) scanning has facilitated in making an early diagnosis and reducing mortality associated with $\mathrm{EDH}^{5,6}$ In a well-organized neurosurgical center, an excellent outcome should be achieved, and "zero mortality" would seem a fair target. Accordingly, a good outcome has been seen in patients treated for $\mathrm{EDH}$ at our center.

\section{METHODS}

We retrospectively reviewed all traumatic $\mathrm{EDH}$ cases seen in the specialized pediatric neurosurgery setting of Kota Kinabalu from January 2013 to August 2018. Patients' case records, operative notes and radiological images were tracked and reviewed. A total of 101 cases were identified. In this series, all indicated patients underwent a CT brain scan that was inclusive of a bone window and a non-contrastenhanced brain parenchyma setting. Only patients where the scans confirmed the presence of an $\mathrm{EDH}$ were included.

The first clinical examination was made in the casualty unit; neurological examinations, pupillary size and reflexes, Glasgow Coma Scale (GCS) scores of the patient at the time of examination by neurosurgical trained personnel, age, gender, mode of injury, site and thickness of the EDH and Glasgow Outcome Scale (GOS) were analyzed. 
These patients were admitted to the neurosurgical unit for close observation of their GCS, pupillary sizes and reflexes. Any new onset or worsening symptoms of increased intracranial pressure such as headaches, vomiting, seizures, neurological deficits were observed. Patients with significant radiological findings, with or without clinical

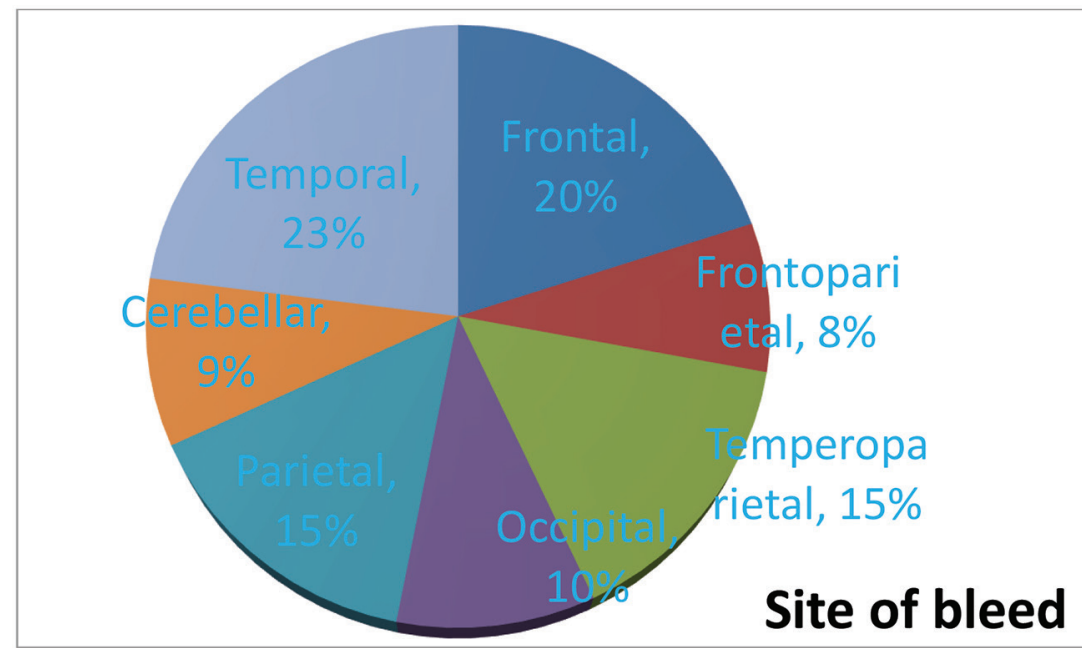

Figure 1. Site of bleed

$70 \%$ $61 \%$

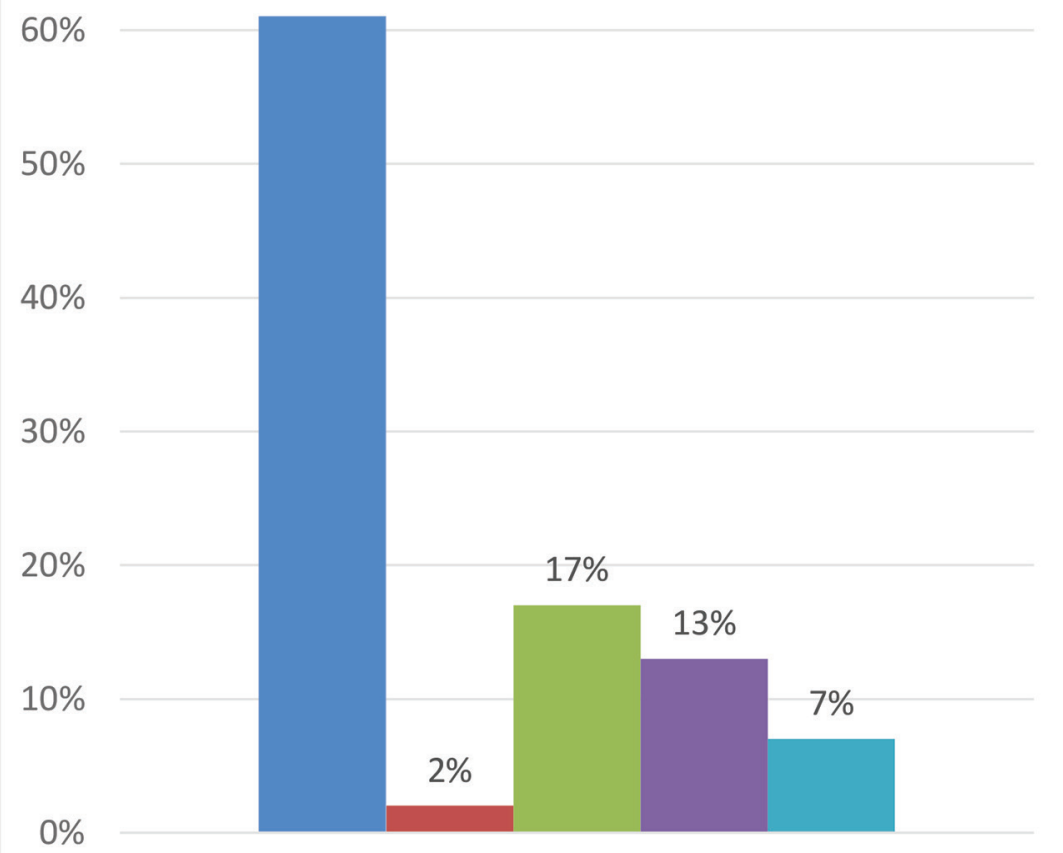

At 6 months

GOS 5 GOS 3 - District $\square$ Defaulted $\square$ Lost to follow up symptoms, were selected for surgical intervention while the rest were managed symptomatically.

\section{RESULTS}

We had 101 patients ranging from the age of 9 months to 12 years. From 101 patients, 58 (58.41\%) were males, and 42 were females (41.59\%). A total of 37 patients (36.3\%) were treated surgically, and 64 patients $(63.7 \%)$ were treated conservatively. About 92 patients $(91.09 \%)$ presented with supratentorial $\mathrm{EDH}$ and 9 patients $(8.91 \%)$ had infratentorial EDH. Skull fractures were present in 47 children (46.5\%).

In the pediatric age group, fall from heights were the most common cause, followed by motor vehicle accident and others (including assault and hit by falling objects). The most frequent site of bleed was the temporal region, noted in 23 patients (22.72\%), followed by the frontal region in 20 patients, the temporoparietal region in 16 patients, the parietal region in 15 patients, cerebellar in 9 patients and the frontoparietal region in 8 patients (Figure 1). About 12 patients with associated intracranial lesions were also identified, namely: thin subdural hemorrhage, small subarachnoid hemorrhage and small contusional bleeds. Intraoperative sites of bleeding were identified, both bleeding from the fracture sites and dural vessel seem to be equal in number (10 each), while only 1 patient had an arterial origin. Two patients had both bleeding from the fracture site and dural vessel. We achieved zero mortality among our patients who were treated both surgically and conservatively.

Due to incomplete documentation and missing records, only 72 patients were able to be classified into traumatic brain injury scoring based on the GCS; 62 patients had a mild traumatic brain injury, 4 had a moderate traumatic brain injury, and 6 with severe traumatic brain injury. Six patients with unequal pupils were identified and treated surgically. Most of the patients who presented with a mild traumatic brain injury requiring surgery were operated by neurosurgical medical officers (12 cases), while most moderate to severe traumatic brain injury were operated by specialized neurosurgeons (10 cases).

Based on the available records, 69 patients had a GOS of 5 upon discharge, while 2 patients with a GOS of 3 were identified. Both patients who had poor GOS presented to the casualty with severe traumatic brain injury with unequal pupils. Our study also shows that we achieved a GOS 5 for 61 of our patients at 6 months of review and the rest were either seen in our district clinics, lost to follow up or chose to default our follow-ups (Figure 2).

Figure 2. GOS at 6 months of follow-up 


\section{DISCUSSION}

The occurrence of EDH in children is known to be rare as the dura layer strongly adheres to the inner table of the skull as well as the elasticity of the skull. ${ }^{6}$ Although rare, they take up about $1-$ $3 \%$ among all pediatric traumatic brain injury. ${ }^{6-8}$ Before the existence of CT scans, the mortality rate was reported to be as high as $86 \% .{ }^{1}$ The majority of studies conducted after the era of CT scans being widely available have reported mortality as low as $20-12 \%$ for traumatic EDH. ${ }^{1}$

It has been traditionally shown in the literature from years before that all extradural hematoma will need to undergo surgical evacuation for better patient outcomes. ${ }^{4,5}$ Over the years, this notion has been challenged. ${ }^{2}$ Since commencing this center, we were keen to compare our results with that of earlier and similar published series to evaluate our therapeutic protocols with the rest of the world.

The result of this study shows that we have achieved zero mortality both from our surgical management as well as our conservative management, being the gold standard with favorable GOS of 5 in most cases and a GOS of 3 in 2 cases. With the above-described results, we believe that postulating a standard protocol of management can be practiced across the state of Sabah. From our study, we noticed that the main factor indicated for surgical management of patients more often than not pointed towards the clinical status of the patient. The time taken from the initial onset of trauma to indicate surgery were not correlative of the general outcome of the patient, as stated in a previous study. ${ }^{9}$ The median time lapsed was 2 days, with most patients treated symptomatically.

In other series, the relationship between the site of bleed and mortality seem contradictory; Kvarnes and Trumpy reported that the site of EDH did not influence mortality. ${ }^{10}$ However, Mc Laurin et al. reported that temporal hematomas had the worst outcome. ${ }^{11}$ In general, our patients had good outcomes regardless of the site of bleed, except for 2 patient whose bleeds were at the temporal with skull base fracture and mydriasis and another with bleeds at the frontal, temporal, parietal and an associated intracranial SDH, also with mydriasis. Cohen et al. reported $100 \%$ mortality in a series of patients with acute EDH and mydriasis for more than 70 minutes. ${ }^{12}$ Moreover, the presence of mydriasis less than 70 minutes were associated with a GOS of 4 and $5 .{ }^{13}$ In our study, 2 patients had poor GOS; it is highly possible that a multifactorial cause were the contributing factors. ${ }^{4,8}$

Our overall satisfactory results were made possible by the all-out cooperation of doctors from our emergency service and the doctors in the district hospital, who were aware that in order to improve outcome, one must prevent deterioration rather than wait for it to appear. It is also commendable that our center has a 24-hour system for a CT scan available for immediate diagnosis and to treat with the least delay even before the onset of clinical signs.

This study, however, has its limitations. Firstly, this study was done retrospectively. Generally, patients with traumatic brain injury are not followed in the clinic for a long time; therefore, patient details were retrieved mainly from documentations. In most patients, there was a lack of standardized neuropsychological evaluation that has to be considered as part of outcome evaluation in children. Next, a detailed description of radiological findings was not documented. Fundamental radiological description regarding the extent of midline shift, clot volumes may have played an additional role in the outcome of this study. Considering the analysis of the different factors influencing the outcome of this study, we believe that a prospective study is needed in Sabah for further effectiveness in treatment protocols that may benefit neurosurgical centers in other developing countries.

\section{CONCLUSION}

$\mathrm{EDH}$ is a neurotrauma emergency that can be lethal, yet it is the most rewardingly responsive lesions treated by neurosurgeons. Early diagnosis, prompt referrals, early surgical intervention when indicated, and good intensive care remain the paramount facets towards achieving zero mortality in EDH. This study proves that our results are comparable to those seen in other neurosurgical centers throughout the developed world. An institutional protocol for EDH may ensure that the ultimate goal of towards 'zero mortality' could be achieved systematically and consistently.

\section{CONFLICT OF INTEREST}

None declared

\section{FUNDING}

No funding was provided for this manuscript.

\section{AUTHOR'S CONTRIBUTION}

All authors equally contributed to the manuscript preparation, manuscript editing, and final manuscript.

\section{REFERENCES:}

1. Bricolo AP, Pasut LM. Extradural hematoma: Toward zero mortality. A prospective study. Neurosurgery. 1984; 14(1): 
8 - 12. DOI: $10.1227 / 00006123-198401000-00003$.

2. Ciurea AV, Kapsalaki EZ, Coman TC, Roberts JL, Robinson 3rd JS, Tascu A, et al. Supratentorial epidural hematoma of traumatic etiology in infants. Childs Nerv Syst. 2007; 23(3): 335 - 41. DOI: 10.1007/s00381-006-0230-4.

3. Jamieson KG, Yelland JDN. Extradural Hematoma. J Neurosurg. 1968; 29(1): 13 - 23.

4. Rocchi G, Caroli E, Raco A, Salvati M, Delfini R. Traumatic epidural hematoma in children. J Child Neurol. 2005; 20(7): 569 - 72. DOI: 10.1177/08830738050200070501.

5. Cheung PSY, Lam JMY, Yeung JHH, Graham CA, Rainer TH. Outcome of traumatic extradural haematoma in Hong Kong. Injury. 2007; 38(1): 76 - 80. DOI: 10.1016/j. injury.2006.08.059.

6. Jung S-W, Kim D-W. Our experience with surgically treated epidural hematomas in children. J Korean Neurosurg Soc. 2012; 51(4): 215 - 8. DOI: 10.3340/jkns.2012.51.4.215.

7. Jamjoom A, Cummins B, Jamjoom ZA. Clinical characteristics of traumatic extradural hematoma: A comparison between children and adults. Neurosurg Rev. 1994; 17(4): 277 - 81. DOI: 10.1007/bf00306818.

8. Dhellemmes P, Lejeune JP, Christiaens JL, Combelles
G. Traumatic extradural hematomas in infancy and childhood. J Neurosurg. 1985; 62(6): 861 - 4. DOI: 10.3171/ jns.1985.62.6.0861.

9. Baykaner K, Alp H, Çeviker N, Keskil S, Seçkin Z. Observation of 95 patients with extradural hematoma and review of the literature. Surg Neurol. 1988; 30(5): 339 - 41. DOI: 10.1016/0090-3019(88)90195-4.

10. Kvarnes TL, Trumpy JH. Extradural haematoma. Acta Neurochir. 1978; 41(1): 223 - 31.

11. Robert LM, Lowell EF. Extradural Hematoma. J Neurosurg. 1964; 21(5): $364-71$.

12. Ingraham FD, Campbell JB, Cohen J. Extradural hematoma in infancy and childhood. J Am Med Assoc. 1949; 140(12): $1010-3$.

13. Erşahin Y, Mutluer S, Güzelbag E. Extradural hematoma: analysis of 146 cases. Childs Nerv Syst. 1993; 9(2): 96 - 9. DOI: $10.1007 / \mathrm{bf00305316.}$

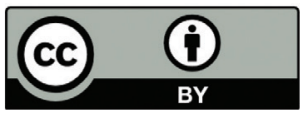

This work is licensed under a Creative Commons Attribution 